\title{
Assessing Self-Reported Adherence to HIV Therapy by Questionnaire: The SERAD (Self-Reported Adherence) Study
}

\author{
JOSE A. MUÑOZ-MORENO, ${ }^{1,2,3}$ CARMINA R. FUMAZ, ${ }^{1,2,3}$ MARIA J. FERRER, ${ }^{2,3}$ ALBERT TULDRÀ, ${ }^{1,2,3}$ \\ TATIANA ROVIRA, ${ }^{3}$ CARME VILADRICH,${ }^{3}$ RAMON BAYÉS,${ }^{3}$ DAVID M. BURGER,${ }^{4}$ \\ EUGÈNIA NEGREDO, ${ }^{1,2,3}$ BONAVENTURA CLOTET, ${ }^{1,2,3,5}$ and the SERAD VALIDATION TEAM*
}

\begin{abstract}
The relationship between adherence to highly active antiretroviral therapy (HAART) and RNA-HIV viral load outcomes has been extensively shown. Although there are different procedures for assessing treatment adherence, there is no ideal method. We present the SERAD (Self-Reported Adherence) questionnaire, a qualitative and quantitative self-reported instrument designed to provide an easier adherence measurement. We also compared the questionnaire to three other methods to evaluate adherence to HAART regimens in HIVinfected patients. Two prospective, observational, longitudinal studies were developed: a single-center pilot study followed by a multicenter study. A total of $530 \mathrm{HIV}$-infected outpatients was prospectively included, 66 in the pilot study and 464 in the multicenter study. Four methods were used to study adherence to HAART regimens: the SERAD questionnaire, pill count, electronic monitoring, and plasma drug monitoring. Pearson's correlations and Bland and Altman's method were developed. The SERAD questionnaire showed good feasibility and significant validity. Adequate levels of agreement between methods were observed, particularly when adherence was high. Differences increased as adherence fell. Moreover, the questionnaire was completed correctly, the interviewers did not report uncovered aspects, and the information was collected easily. Our results suggest that the SERAD questionnaire is a feasible and useful instrument for assessing adherence to HAART regimens in HIV-infected patients, and makes it possible to obtain reliable qualitative and quantitative information related to treatment adherence.
\end{abstract}

\section{INTRODUCTION}

A DHEREnCE TO Highly Active Antiretroviral Therapy (HAART) has been extensively related to RNA-HIV viral load outcomes and this has confirmed the strong relationship between both issues. ${ }^{1-5}$ For this reason, an optimal knowledge of adherence to antiretroviral treatment appears to be relevant for clinical decisions such as HAART simplification or intensification, or interrupting or delaying treatment. ${ }^{6}$ Moreover, reliable adherence-related information may help clinicians to ascertain the difficulties encountered in taking the medication adequately. ${ }^{7}$
Despite of the need for a rigorous assessment of HAART adherence, a "gold standard" has not yet been found. ${ }^{8}$ Hitherto, the most commonly used methods to evaluate HAART adherence have been pill count, plasma drug monitoring, electronic monitoring, and patient self-reporting. However, although some studies have used these different assessment methods, not much information is available with respect to comparisons among them. ${ }^{7}$

The most usual, feasible, and less expensive way to assess HAART adherence has been patient self-reporting. Nevertheless, this method has been criticized for methodological reasons. ${ }^{9}$ Its validity may be limited by the type of questions used,

\footnotetext{
${ }^{1}$ Lluita contra la SIDA Foundation, Badalona, Barcelona, Catalonia, Spain.

${ }^{2}$ Germans Trias i Pujol University Hospital, HIV Unit, Badalona, Barcelona, Catalonia, Spain.

${ }^{3}$ Autònoma de Barcelona University, Barcelona, Catalonia, Spain.

${ }^{4}$ University Hospital of Nijmegen, Nijmegen, The Netherlands.

${ }^{5}$ IrsiCaixa Foundation, Badalona, Barcelona, Catalonia, Spain.

*The members of the SERAD Validation Team are listed in the Acknowledgments.
} 
question style, or the time setting in which the question is asked. Similarly, self-reported adherence is further characterized by the need for optimal psychometric properties. And though some questionnaires to assess self-reported HAART adherence have been proposed, the qualitative and quantitative information provided by them has been limited. ${ }^{9}$ For these reasons, there is a need to find self-report instruments with precise accuracy for antiretroviral therapy adherence assessment.

In this study we developed a standardized self-reported questionnaire, the SERAD (Self-Reported Adherence) questionnaire, which was designed to provide significant qualitative and quantitative information related to HAART adherence. Its feasibility, validity, and usefulness were studied in a pilot and multicenter study and, in addition, the application of the SERAD was compared to three other relevant adherence methods: pill count, electronic monitoring, and plasma drug monitoring.

\section{MATERIALS AND METHODS}

\section{Patients}

A total of 11 Spanish hospitals participated in the investigation (see Acknowledgments). HIV-infected outpatients aged $\geq 18$ years old and taking a HAART combination were invited to participate in the study. HAART combination was defined as an antiretroviral treatment including two nucleoside or nucleotide reverse transcriptase inhibitors + one nonnucleoside reverse transcriptase inhibitor, or two nucleoside or nucleotide reverse transcriptase inhibitors + one or more protease inhibitor \pm one fusion inhibitor, or one nonnucleoside reverse transcriptase inhibitor + one protease inhibitor. Patients were informed of the purpose and procedures of the investigation, and those who agreed to participate were recruited. All participants provided written informed consent.

\section{Study design}

Two prospective, observational, comparative studies were designed, consisting of a pilot study followed by a multicenter study. The local ethics committee approved both study protocols.

Pilot study. A preliminary study was conducted to test the feasibility of the subsequent multicenter protocol. Only patients from the HIV Unit of the Germans Trias i Pujol University Hospital (Barcelona, Spain) took part in this first single-center study. Research subjects were followed up for 2 months and assessed at visits at week 0 (baseline evaluation), week 4 (month 1 ), and week 8 (month 2). Sociodemographic, clinical, and psychological variables were collected. Four methods were used to study individual adherence: the SERAD questionnaire, pill count, electronic monitoring, and plasma drug monitoring.

Multicenter study. Following the completion of the pilot study and the analysis of the results, a second protocol was developed. This consisted of a multicenter study that maintained the first study design. The only difference was the inclusion of a new follow-up visit at week 12 (month 3), which was established to offer the possibility of covering a longer period of time to assess adherence. The 11 aforementioned HIV units participated.

\section{Medical and psychological assessment}

The baseline evaluation included the following demographic and clinical variables: age, gender, HIV infection route, current consumption of toxic substances, time since $\mathrm{HIV}^{+}$diagnosis, current $\mathrm{CD}^{+}{ }^{+}$cell count and percentage, viral load, current HAART and usual schedule, weeks on current treatment, and antiretroviral treatment history. The information was collected from patients' medical records, reviewed, and completed at each clinical visit. At the baseline interview, perceived social support for HIV infection and perceived capacity to follow treatment (self-efficacy) were measured on a 100 -mm visual analogue scale (VAS). ${ }^{10}$ An instrument previously used in HIV populations, the Beck Depression Inventory, ${ }^{11}$ was applied to assess depression at the baseline visit. The $16 \mathrm{PF}-5$ Inventory ${ }^{12}$ was used during the first month of follow-up. This tool is one of the most commonly used personality assessment methods, and was completed by patients to control a possible bias in the responses provided by subjects to fulfill the interviewer's expectations. ${ }^{13}$ The three-style response indices provided by this instrument, impression management (IM), infrequency (IN), and acquiescence (AQ) scales, were added as variables to be controlled in data statistical analyses.

\section{Adherence assessment}

SERAD questionnaire. Since hitherto there has been no one reliable way of measuring HAART adherence, we decided to create an easy, fast, and comfortable instrument that also provided reliable qualitative and quantitative information in a simple way.

The SERAD (Self-Reported Adherence) questionnaire ${ }^{14}$ was originally developed in Spanish, although it has now been translated into English (all versions are available at www.flsida.org/serad). The Spanish version was used in the SERAD study.

This tool was designed to be an interviewer-administered questionnaire to ensure greater validity of the data obtained. Most of the questions in the questionnaire were open, focusing on obtaining quantitative answers. A SERAD usage guide ${ }^{14}$ was created to facilitate the administration by the clinician.

Three main parts were considered in the formulation of the SERAD. The initial part collected information on the patient's medication posology. This part was regarded as initial and basic, and more specifically included the medication prescribed, the schedule to be followed, the number of dosage units prescribed, and the number of daily doses. The second part dealt with the adherence data. Unlike other self-reported methods of adherence assessment, the SERAD provided for two types of indexes. One was based on the number of times the patient failed to take the medication (adherence percentage) and the other one on the times the patient failed to observe the intake conditions (intake conditions observed percentage). Thus, the questionnaire made it possible to assess the number of times the patient did not take the medication, and the compliance with the time conditions related to the treatment, another particularly important factor in the clinical setting.

The time-specific contexts covered by the questionnaire referred to the last week, the last month, and the last 3 months. The possibility of covering three time contexts was a major advantage envisaged from the outset of the design. These times 
were chosen to provide structured and sufficiently representative information in terms of time. The measurement of 1 day prior to the application of the questionnaire was ruled out as it was deemed to be rather unrepresentative. On the other hand, some studies use measurements pertaining to 3 or 4 days before the questionnaire, ${ }^{10,15,16}$ although we considered that it was easier for the patient to remember 7 days, which is one week, rather than 4. Moreover, because some studies had shown good results with these three time settings, ${ }^{17}$ the decision was finally taken to use these three measurements.

The reasons for nonadherence were also recorded whenever noncompliant behavior was mentioned because of the evident clinical importance. They were coded in 13 items chosen from the most frequently reported ones in clinical practice. ${ }^{18}$ This was one of the main questions addressed because of the evident clinical importance of knowing why patients fail to follow treatment properly.

The third part of the design included the totals of the calculations and the final results. The percentages of adherence were calculated on the basis of the following formula: (total number of dosage units prescribed - total number of times reported)/(total number of dosage units prescribed $) \times 100$. And the percentages with regard to the reasons for nonadherence were calculated as follows: (total number of reasons reported total number of one concrete reason reported)/(total number of reasons reported) $\times 100$. In the end, the SERAD made it possible to calculate the following outputs:

1. Percentage of adherence with regard to the doses missed during the last week.

2. Percentage of reasons for nonadherence during the last week.

3. Percentage of adherence with regard to the times that intake conditions were not observed during the last week.

4. Percentage of reasons for intake conditions not observed during the last week.

5. Percentage of adherence with regard to the doses missed during the last month.

6. Percentage of reasons for nonadherence during the last month.

7. Percentage of adherence with regard to the times that intake conditions were not observed during the last month.

8. Percentage of reasons for intake conditions not observed during the last month.

9. Percentage of adherence with regard to the doses missed during the last 3 months.

From the outset, our aim was to generate a single-sheet questionnaire in a comfortable format. Finally, the questionnaire comprised a single sheet, and a computerized data base was created where the results were calculated automatically.

To test its feasibility, the time to complete the questionnaire was recorded and possible mistakes were also considered. The latter were defined as any information recorded on the questionnaire that would render the correction process impossible. The correction process consisted of the calculation of the adherence percentages as well as the calculation of the rest of the quantitative information provided by the SERAD. At the end of the pilot and multicenter study the investigators were questioned concerning the difficulty of completing the questionnaire and possible relevant suggestions to improve data col- lection. The SERAD was applied at week 4, 8 (pilot and multicenter study), and 12 (multicenter study) of the patient follow-up.

Pill count. After baseline visit, patients collected their HAART regimen from the pharmacy hospital service, where the number of dosage units dispensed was recorded. Patients were instructed to return only the surplus medication collected for the month at the next visit. This medication was recorded again. It was assumed that patients consumed the medication that was not returned. However, subjects reporting the use of prior medication, or medication not returned by mistake or forgetfulness, were not included in the evaluation. On the basis of number of dosage units returned, an adherence percentage was calculated with regard to the doses missed during the last month. The following formula was applied: (total number of dosage units collected - total number of dosage units returned)/(total number of dosage units collected) $\times 100$. This adherence assessment method was applied at the same visits as the SERAD.

Electronic monitoring. Electronic drug exposure monitors (eDEM, AARDEX Ltd., Zug, Switzerland; www.aardex.ch) were used. Due to logistics and cost, only $10 \%$ of patients in the multicenter study participated. The recruitment of patients was conducted according to a computer-generated randomnumber table. The subjects selected were offered the possibility of training in the use of electronic monitoring. If they declined, another patient was assigned and proposed with regard to the randomization table. Patients who accepted were provided with an electronic drug exposure monitor cap bottle for one of their antiretroviral drugs. Each drug assigned in the bottles was selected with regard to a previously randomized list of antiretroviral drugs, established according to the criterion of the pharmacists of the hospital pharmacy service. The drugs were selected particularly on the basis of dosage units that were easiest to store in this type of container. The software incorporated in the cap bottles (provided by AARDEX Ltd.) reported the number of days monitored, the number of doses taken, adherence percentage, percentage of days on which the doses prescribed were taken correctly, and the percentage of prescribed doses taken on schedule (intake conditions observed percentage). The time range considered was $2 \mathrm{~h}$ before and after the ingested dose. Patients were specifically instructed to open the electronic monitor cap bottle only when they took the medication, and if for any reason they opened the bottle off-schedule they were to record this and provide the information at the following visit. Patients were provided with a refilled bottle at baseline, week 4 , and week 8 .

Plasma drug monitoring. NNRTI and PI concentrations in plasma were assessed in the first 200 patients who were consecutively included in the study. Logistics and cost determined this proportion of patients. In an attempt to avoid modifying the usual adherence to antiretroviral therapy, subjects were not informed that drug concentrations were going to be measured until blood sampling. Blood samples were collected in potassium and ethylenediaminetetraacetic acid (EDTA)-containing $10-\mathrm{ml}$ tubes. Plasma was isolated by centrifugation $(4000 \mathrm{rpm}$ for $15 \mathrm{~min}$ ) and stored at $-80^{\circ} \mathrm{C}$ until analysis. Drug concentrations were determined by high-performance liquid chro- 
matography (HPLC) according to a validated method. ${ }^{19}$ Patients harboring drug concentrations lower than $0.01 \mathrm{mg} / \mathrm{liter}$ were considered to be nonadherent to antiretroviral therapy.

\section{Data analysis}

Analyses to establish the psychometric properties of the SERAD questionnaire were developed.

Feasibility considered qualitative and quantitative aspects: time to complete the questionnaire, recorded mistakes, understanding of the interviewer's instructions, simplicity of the data collected, aspects that could be simplified, and aspects not addressed in the questionnaire.

Validity was regarded as the agreement between the four adherence assessment methods throughout the follow-up. Pearson's correlations were developed to study this point. However, since the Pearson correlation is known to underestimate the level of agreement, a graph analysis was used. This consisted of Bland and Altman's method, which compared agreement between measures depending on different levels of adherence showed. ${ }^{20}$ This method made it possible to obtain agreement between two measures by means of a graph considering the mean of the measures and the difference between them. In the graph, points near 0 indicated a high agreement between measures, while points far from 0 indicated disagreement. Since none of the methods could be considered as a "gold standard," all four methods were compared: SERAD questionnaire, pill count, electronic monitoring, and plasma drug monitoring. Although one of the greatest advantages of SERAD was the possibility of using different adherence measures, only four of them were used in this study. Doses missed during the last week (1) and the last month (5) and intake conditions not respected during the last week (3) and last month (7) were regarded as the most significant adherences measures. Therefore, only these were used for the data analysis. This was because SERAD made it possible to find new adherence measures, although this was not a primary endpoint of the study. And because SERAD was compared with the other three adherence methods to test its feasibility, validity, and usefulness, they did not provide the new outcomes obtained by SERAD.
A

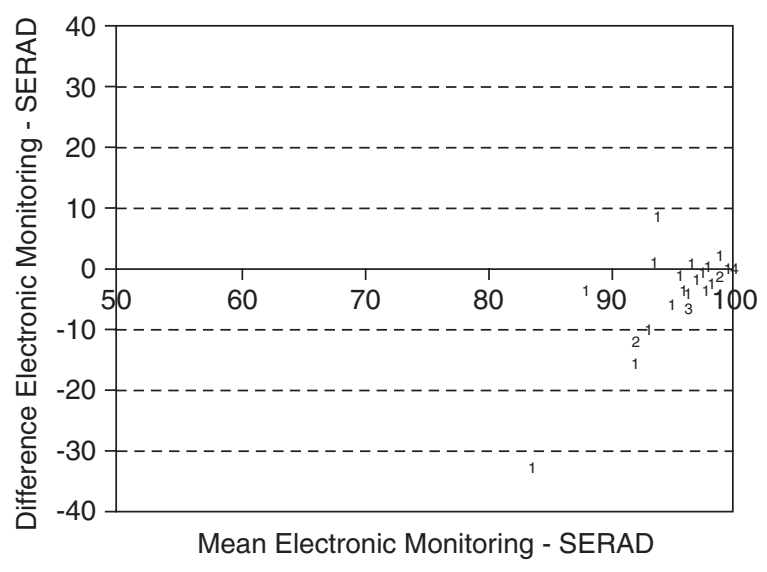

C

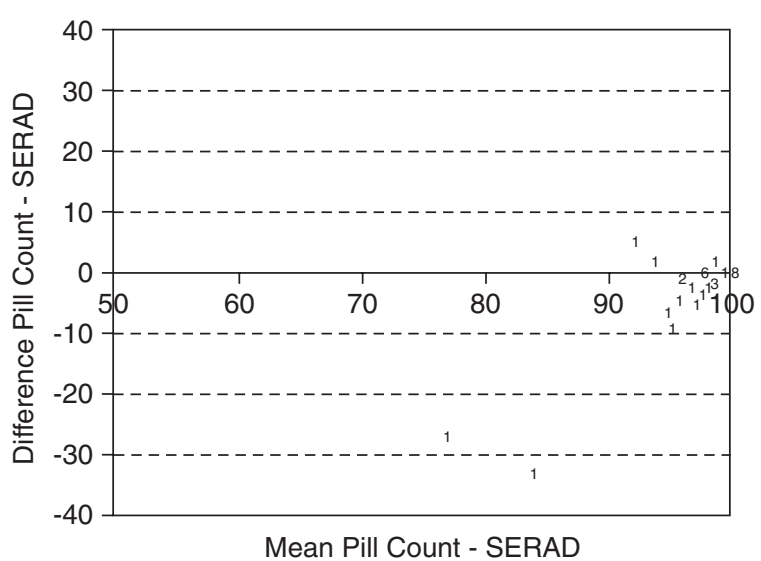

B

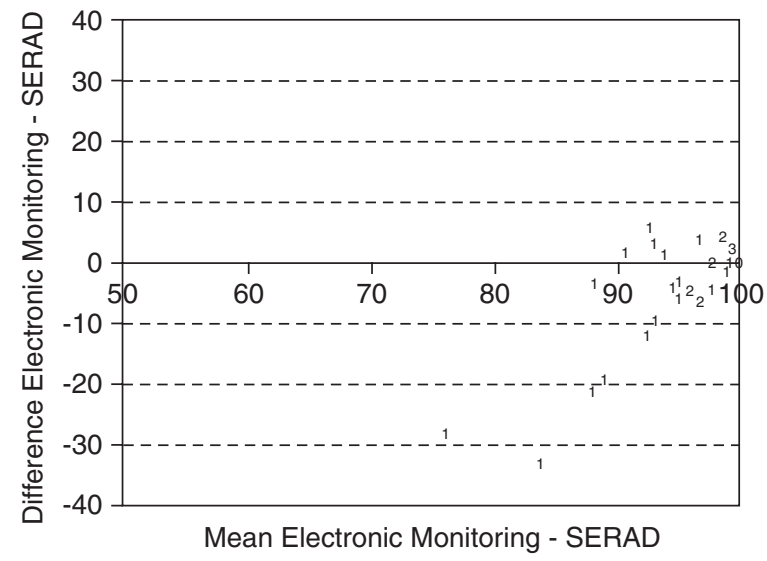

D

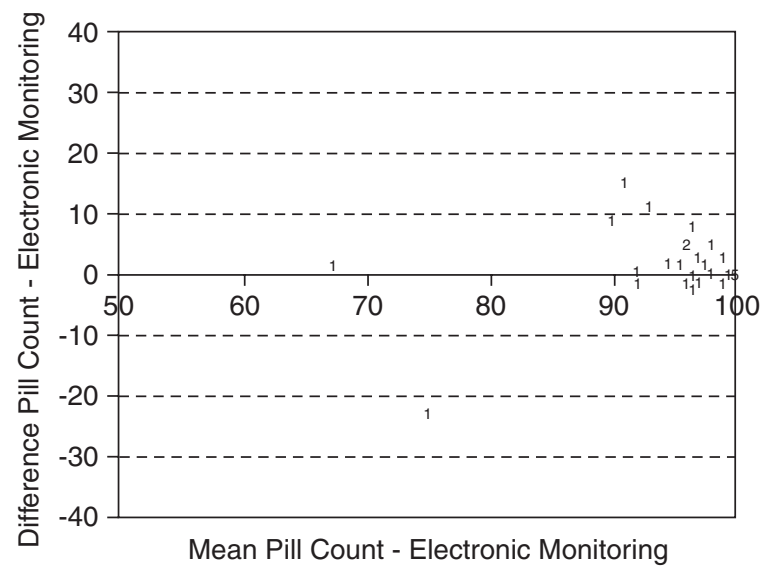

FIG. 1. Comparisons of adherence measures in pilot study by Bland and Altman's method. (A) Agreement between electronic monitoring and SERAD in last month adherence measure $(n=38)$. (B) Agreement between electronic monitoring and SERAD in last month intake conditions measure $(n=38)$. (C) Agreement between pill count and SERAD in last month adherence measure $(n=41)$. (D) Agreement between pill count and electronic monitoring in last month adherence measure $(n=37)$. 
A complementary analysis was performed to convert quantitative adherence methods into qualitative binomial measures. The $95 \%$ adherence was taken as the cutoff point considering the clinical criteria established by Paterson et al. ${ }^{1}$ Thus, noncompliant subjects were those with levels of adherence $<95 \%$, whereas those with levels of adherence $\geq 95 \%$ were regarded as compliant. The qualitative transformation of these adherence measures was used to assess agreement with plasma drug levels.

Since it was thought that differences among adherence methods might appear, although not only associated with adherence outcomes, a logistic regression analysis was performed to control the possible existence of concrete profiles on patients' responses. Moreover, to study this, patients with a $>5 \%$ difference between methods were analyzed by logistic regression. The following variables were included in the analysis: participant center, age, gender, HIV infection route, current consumption of toxic substances, years since $\mathrm{HIV}^{+}$diagnosis, current $\mathrm{CD} 4^{+}$cell count and percentage, current HAART and usual schedule, perceived social support, perceived capacity to follow treatment (self-efficacy), depression, and the three response style indices of the 16PF-5 Inventory (IM, IN, and AQ scales).

\section{RESULTS}

\section{Pilot study}

A total of 66 patients were included in the pilot study. Fortyfive $(68 \%)$ of them were men. The mean $( \pm$ standard deviation) age was $37( \pm 9)$ years with $7( \pm 3)$ years of HIV infection. The use of intravenous drugs was the infection route in 27 (41\%) patients, $16(24 \%)$ heterosexual contact, 12 (18\%) men who had sex with men and $2(3 \%)$ blood product transfusion. Nine $(14 \%)$ patients provided no information about their infection route.

None of the questionnaires presented limitations for correc- tion. They were completed adequately and the correction process of the SERAD was developed as expected. The interviewers did not report aspects not covered in the questionnaire and no investigators stated that they had encountered any difficulties collecting the information. The mean time taken to complete the information was $3.8( \pm 1.8) \mathrm{min}$ at month 1 and $3.3( \pm 1.7)$ at month 2 .

Bland and Altman's method showed that high agreement levels were found, particularly when adherence percentages were high. This fact was observed with regard to the comparison between the SERAD and electronic monitoring, the SERAD and pill count, and electronic monitoring and pill count. Bland and Altman's method for month 1 is shown in Fig. 1. The best levels of agreement were found between the SERAD and electronic monitoring (75\%) and between the SERAD and pill count (71\%). Differences between methods increased when adherence fell.

After considering the data obtained, the design of the questionnaire was maintained for its validation in the multicenter study.

\section{Multicenter study}

A total of 489 patients were informed about the purposes and procedure of the study, and $464(95 \%)$ agreed to participate and gave their written consent. Sixty-seven percent of the patients were men, with a mean age of $38( \pm 10)$ years. The infection route was intravenous drug use in $33 \%$ of patients, $32 \%$ heterosexual intercourse, $23 \%$ men having sex with men, and 5\% blood product transfusion. No information was given by the remaining $7 \%$. The mean years of infection were $8( \pm 5)$. The rest of demographic, clinical, and psychological information is displayed at Table 1.

In the course of the study, 986 SERAD questionnaire forms were obtained $(393,343$, and 250 at months 1,2 , and 3 , respectively). The total mean time to complete the SERAD was $3.7( \pm 3.2) \mathrm{min}$, and mistakes were detected in $2.4 \%$ of the to-

Table 1. Baseline Demographic and Clinical Characteristics of Study Participants ${ }^{\mathrm{a}}$

\begin{tabular}{lc}
\hline Sex (male) & $305(67 \%)$ \\
Age (years) & $38( \pm 10)$ \\
Infection route & \\
$\quad$ Injected drug user & $33 \%$ \\
Heterosexual & $32 \%$ \\
Homosexual & $23 \%$ \\
Blood transfusion & $5 \%$ \\
$\quad$ Unknown & $7 \%$ \\
Time since HIV diagnosis (years) & $8( \pm 5)$ \\
Number of patients reporting toxic substances consumption & $220(47 \%)$ \\
CD4 cell count (cells/ml) & $497( \pm 353)$ \\
$\%$ CD4 cell count (cells/ml) & $27( \pm 49)$ \\
Viral load (log $)$ & $4.3( \pm 5)$ \\
Time on treatment (weeks) & $59( \pm 62)$ \\
Perceived social support (VAS) & $81( \pm 31)$ \\
Perceived self-efficacy (VAS) & $94( \pm 13)$ \\
Depression (BDI) & $11( \pm 9)$ \\
\hline
\end{tabular}

aData expressed as mean values ( \pm standard deviation), except when specified. VAS, visual analogue scale; BDI, Beck depression inventory. 
Table 2. Percentages of Adherence and Intake Conditions Observed, According to the SERAD and Pill Count

\begin{tabular}{|c|c|c|c|c|c|c|}
\hline Month & Method & Period & Measure & $\mathrm{N}$ & $\operatorname{Mean}( \pm S D)$ & Min-Max \\
\hline \multirow[t]{5}{*}{1} & \multirow[t]{4}{*}{ SERAD } & \multirow[t]{2}{*}{ Week } & Adherence & 373 & $97.7( \pm 7.6)$ & $0-100$ \\
\hline & & & Intake cond. & 372 & $97.7( \pm 9.4)$ & $0-100$ \\
\hline & & \multirow[t]{2}{*}{ Month } & Adherence & 373 & $94.4( \pm 12.4)$ & $0-100$ \\
\hline & & & Intake cond. & 372 & $98.3( \pm 6.0)$ & $13-100$ \\
\hline & Pill count & Month & Adherence & 236 & $91.3( \pm 17.5)$ & 0-100 \\
\hline \multirow[t]{5}{*}{2} & \multirow[t]{4}{*}{ SERAD } & \multirow[t]{2}{*}{ Week } & Adherence & 323 & $98.2( \pm 5.9)$ & $28.6-100$ \\
\hline & & & Intake cond. & 323 & $98.3( \pm 8.0)$ & 0-100 \\
\hline & & \multirow[t]{2}{*}{ Month } & Adherence & 323 & $94.4( \pm 11.3)$ & $14.3-100$ \\
\hline & & & Intake cond. & 323 & $98.5( \pm 6.8)$ & $20.4-100$ \\
\hline & Pill count & Month & Adherence & 210 & $88.5( \pm 19.5)$ & $0.0-100$ \\
\hline \multirow[t]{5}{*}{3} & \multirow[t]{4}{*}{ SERAD } & \multirow[t]{2}{*}{ Week } & Adherence & 232 & $98.6( \pm 3.9)$ & $74.3-100$ \\
\hline & & & Intake cond. & 232 & $98.5( \pm 6.5)$ & $28.6-100$ \\
\hline & & \multirow[t]{2}{*}{ Month } & Adherence & 232 & $96.6( \pm 7.1)$ & $60.7-100$ \\
\hline & & & Intake cond. & 232 & $99.1( \pm 2.8)$ & $73.3-100$ \\
\hline & Pill count & Month & Adherence & 138 & $84.7( \pm 22.7)$ & $0.0-100$ \\
\hline
\end{tabular}

tal forms completed. All evaluators considered the questionnaire easy to complete and no significant suggestions to improve it were received.

As far as pill count and electronic monitoring are concerned, $633(253,229,151)$ and $100(38,32,30)$ forms were recorded, respectively. Table 2 shows results from the SERAD and the pill count at each visit, and Table 3 shows the same measures regarding electronic monitoring.
To study the comparison between both of the main time settings considered in the study, adherence over the last week and the last month, a subanalysis was developed with regard to the comparison between all the study participants assessed by the SERAD or pill count or electronic monitoring, and the subgroup of those participants that was electronically monitored. When last-week and last-month adherence percentages were compared, the greatest differences in agreement observed be-

Table 3. Percentages of Adherence and Intake Conditions Observed, According to the SERAD, Electronic Monitoring, and Pill Count for Patients Monitored Electronically

\begin{tabular}{|c|c|c|c|c|c|c|}
\hline Month & Method & Period & Measure & $\mathrm{N}$ & Mean $( \pm S D)$ & Min-Max \\
\hline \multirow[t]{9}{*}{1} & \multirow[t]{4}{*}{ SERAD } & \multirow[t]{2}{*}{ Week } & Adherence & 33 & $93.7( \pm 17.8)$ & 0-100 \\
\hline & & & Intake cond. & 32 & $94.3( \pm 16.4)$ & $16.6-100$ \\
\hline & & \multirow[t]{2}{*}{ Month } & Adherence & 33 & $89.6( \pm 20.8)$ & 0-100 \\
\hline & & & Intake cond. & 32 & $94.1( \pm 16.3)$ & $20-100$ \\
\hline & \multirow[t]{4}{*}{$\mathrm{EM}^{\mathrm{a}}$} & Week & Adherence & 38 & $89.7( \pm 21.8)$ & $0-100$ \\
\hline & & Week & Intake cond. & 37 & $85.7( \pm 16.9)$ & $35.7-100$ \\
\hline & & Month & Adherence & 38 & $91.8( \pm 16.7)$ & $4.5-100$ \\
\hline & & Month & Intake cond. & 38 & $85.9( \pm 15.3)$ & $49.1-100$ \\
\hline & Pill count & Month & Adherence & 34 & $93.9( \pm 16.5)$ & $3.1-100$ \\
\hline \multirow[t]{9}{*}{2} & \multirow{4}{*}{ SERAD } & Week & Adherence & 32 & $98.6( \pm 3)$ & $90.5-100$ \\
\hline & & Week & Intake cond. & 32 & $96.9( \pm 9.1)$ & $50-100$ \\
\hline & & Month & Adherence & 32 & $94.9( \pm 8.9)$ & $57.1-100$ \\
\hline & & Month & Intake cond. & 32 & $99.0( \pm 2.3)$ & $88.3-100$ \\
\hline & \multirow[t]{4}{*}{ EM } & Week & Adherence & 32 & $94.0( \pm 11.7)$ & $57.1-100$ \\
\hline & & Week & Intake cond. & 32 & $85.7( \pm 16.9)$ & $42.9-100$ \\
\hline & & Month & Adherence & 32 & $93.0( \pm 11.6)$ & $62.2-100$ \\
\hline & & Month & Intake cond. & 32 & $85.1( \pm 15.6)$ & $40-100$ \\
\hline & Pill count & Month & Adherence & 26 & $87.7( \pm 21.9)$ & 0-100 \\
\hline \multirow[t]{9}{*}{3} & \multirow[t]{4}{*}{ SERAD } & Week & Adherence & 27 & $97.3( \pm 4.5)$ & $85.7-100$ \\
\hline & & Week & Intake cond. & 27 & $96.2( \pm 10.3)$ & $53.9-100$ \\
\hline & & Month & Adherence & 27 & $93.1( \pm 11.8)$ & $57.1-100$ \\
\hline & & Month & Intake cond. & 27 & $98.3( \pm 3.8)$ & $85.2-100$ \\
\hline & \multirow[t]{4}{*}{ EM } & Week & Adherence & 30 & $93.5( \pm 15.1)$ & $21.4-100$ \\
\hline & & Week & Intake cond. & 30 & $78.7( \pm 24.9)$ & $0-100$ \\
\hline & & Month & Adherence & 30 & $94.9( \pm 10.5)$ & $46.2-100$ \\
\hline & & Month & Intake cond. & 30 & $79.8( \pm 17.3)$ & $48.1-100$ \\
\hline & Pill count & Month & Adherence & 23 & $90.7( \pm 21.1)$ & 0-100 \\
\hline
\end{tabular}

${ }^{\mathrm{a} E M}$, electronic monitoring. 
A

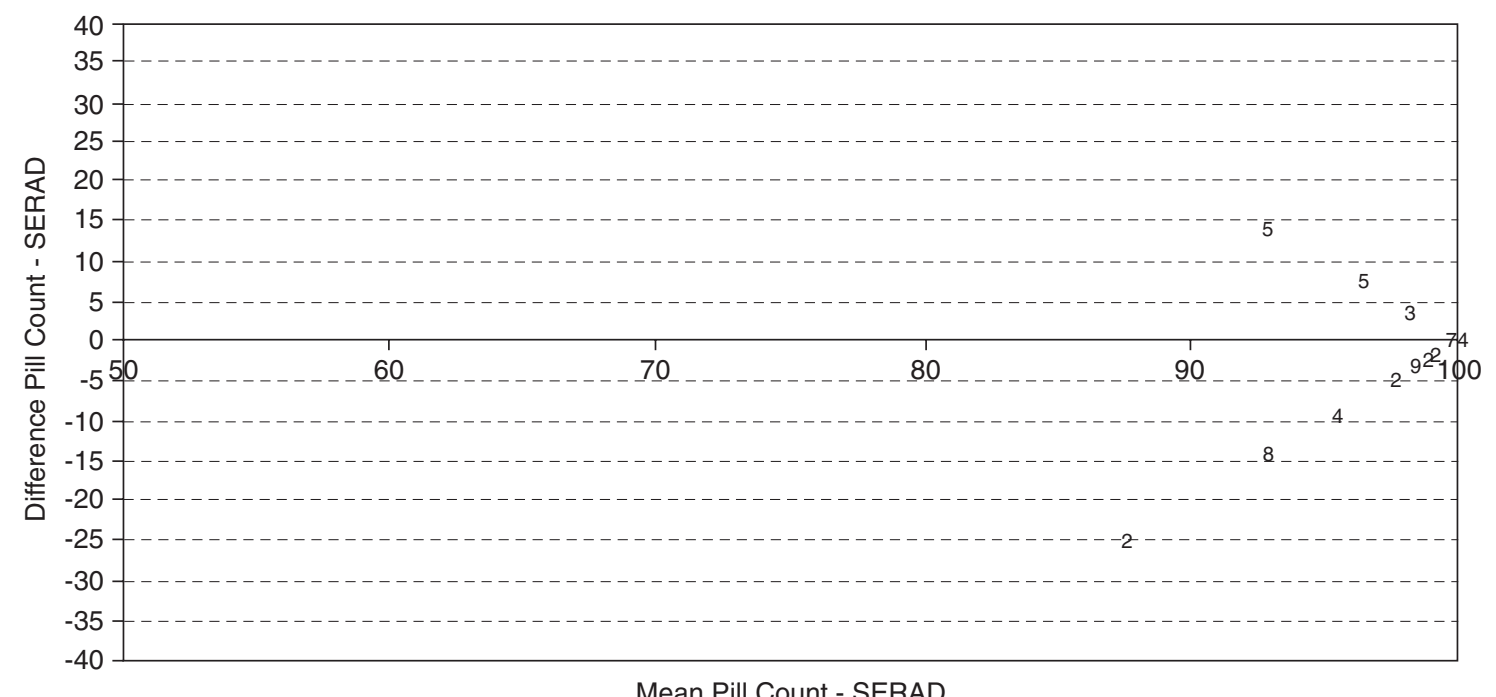

B

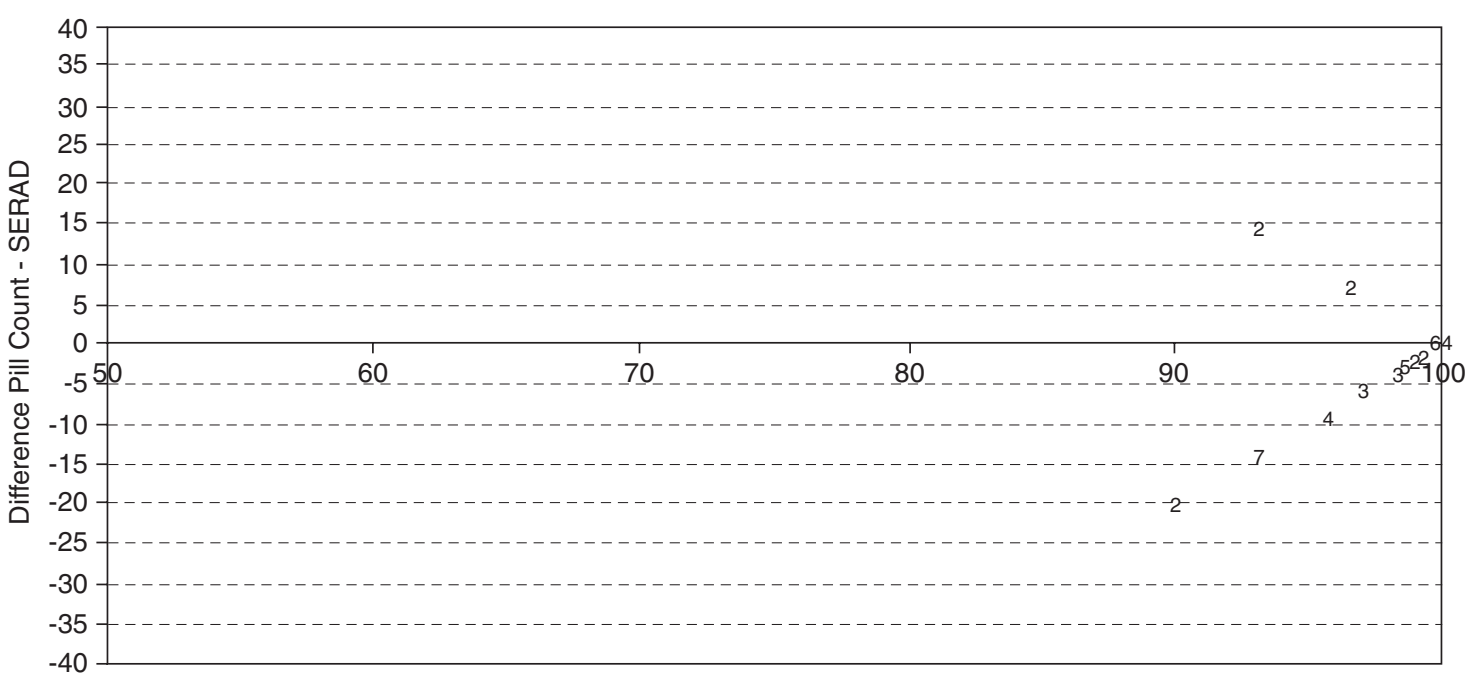

Mean Pill Count - SERAD

C

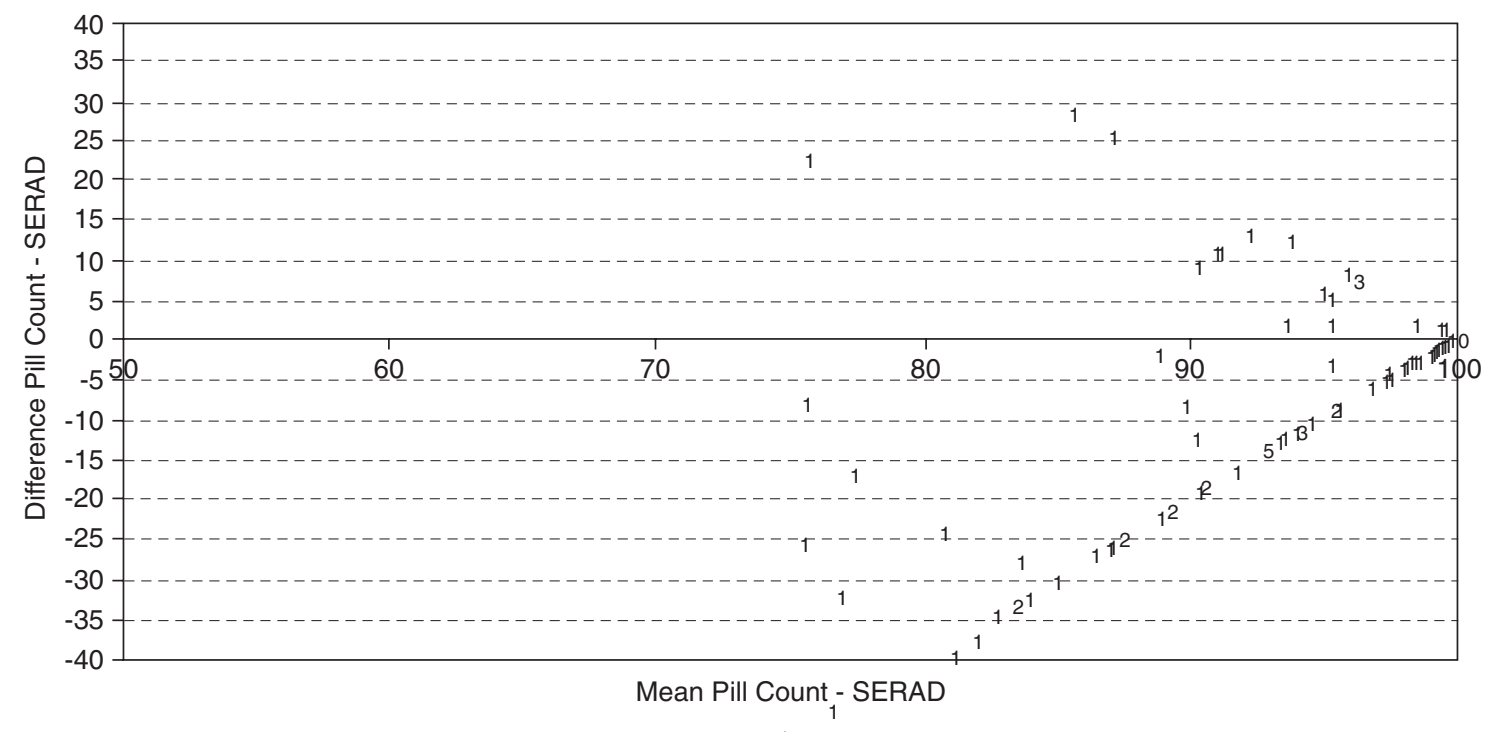


Table 4. Differences among Different Adherence Assessments

\begin{tabular}{|c|c|c|c|c|c|c|}
\hline Month & Method $^{\mathrm{a}}$ & Period & Measure & $\mathrm{n}$ & Mean $( \pm S D)$ & Min-Max \\
\hline \multirow[t]{6}{*}{1} & \multirow[t]{4}{*}{ EM-SE } & \multirow[t]{2}{*}{ Week } & Adherence & 32 & $-3.6( \pm 11.9)$ & $(-50)-14.3$ \\
\hline & & & Intake cond. & 31 & $-9.3( \pm 22.4)$ & $(-60)-66.7$ \\
\hline & & \multirow[t]{2}{*}{ Month } & Adherence & 32 & $1.8( \pm 11.5)$ & $(-20.5)-54.2$ \\
\hline & & & Intake cond. & 31 & $-9.6( \pm 16.5)$ & $(-49.2)-31.7$ \\
\hline & PC-SE & Month & Adherence & 24 & $4.3( \pm 7.55)$ & $(-7.55)-53$ \\
\hline & PC-EM & Month & Adherence & 27 & $0.5( \pm 2.5)$ & $(-3.5)-8.2$ \\
\hline \multirow[t]{6}{*}{2} & \multirow[t]{4}{*}{ EM-SE } & \multirow[t]{2}{*}{ Week } & Adherence & 32 & $-4.6( \pm 10.5)$ & $(-35.7)-7.1$ \\
\hline & & & Intake cond. & 32 & $-11.2( \pm 19.4)$ & $(-57.2)-36.7$ \\
\hline & & \multirow[t]{2}{*}{ Month } & Adherence & 32 & $-1.8( \pm 10.6)$ & $(-37.8)-12.6$ \\
\hline & & & Intake cond. & 32 & $-13.8( \pm 16)$ & $(-60)-5$ \\
\hline & PC-SE & Month & Adherence & 25 & $-6.5( \pm 16.3)$ & $(-57.1)-14.3$ \\
\hline & PC-EM & Month & Adherence & 25 & $-5.3( \pm 16.6)$ & $(-65.7)-29$ \\
\hline \multirow[t]{6}{*}{3} & \multirow[t]{4}{*}{ EM-SE } & \multirow[t]{2}{*}{ Week } & Adherence & 27 & $-4.1( \pm 16.4)$ & $(-78.6)-14.3$ \\
\hline & & & Intake cond. & 27 & $-16.7( \pm 25.8)$ & $(-100)-7.7$ \\
\hline & & \multirow[t]{2}{*}{ Month } & Adherence & 27 & $2.4( \pm 8.7)$ & $(-11)-35.5$ \\
\hline & & & Intake cond. & 27 & $-17.0( \pm 16.7)$ & $(-48)-1.7$ \\
\hline & PC-SE & Month & Adherence & 22 & $-3.7( \pm 14.8)$ & $(-57.1)-11.1$ \\
\hline & PC-EM & Month & Adherence & 23 & $-6.6( \pm 20.2)$ & $(-92.6)-3.9$ \\
\hline
\end{tabular}

${ }^{\mathrm{a} E M}$, electronic monitoring; SE, SERAD; PC, pill count.

tween both measures were $3.7 \%$ in all participants and $4.7 \%$ in the subgroup. When only electronic monitoring assessment was studied, the greatest difference found was $2 \%$. These differences did not present statistical significance.

Logistic regression analysis determined that none of the variables considered showed a significant influence between patients and methods (data not shown). Bland and Altman's analysis made it possible to obtain levels of agreement between different adherence assessment methods, and showed that high levels were observed, particularly when adherence was high. Figure 2 shows the agreement between SERAD and pill count.

When only patients electronically monitored were analyzed, differences between the SERAD and electronic cap bottles measures were less than $3 \%$ comparing adherence in the last month in all cases. Table 2 shows results from the SERAD, pill count, and electronic monitoring at each visit for electronically monitored patients. The comparison between the SERAD and pill count measures made it possible to observe differences obtained in a range between $0.5 \%$ and $6.6 \%$.

When the intake conditions percentage was compared, differences increased in a range of 9-17\%. Bland and Altman's method provided a very similar profile of results for all participants.

Differences between the SERAD, electronic monitoring, and pill count are shown in Table 4.

One hundred and eighty-four samples (40\%) were obtained to determine plasma drug monitoring. Rates of agreement between this assessment method and the others were very high (data not shown).

When the last-week adherence percentage of the SERAD was considered, an agreement was observed in 141 of 166 patients
(85\%). When the last-month adherence percentage was considered it was 119 out of $166(72 \%)$. On the other hand, pill count coincided with plasma drug levels in only 59 of 100 cases $(59 \%)$.

When only patients on electronic monitoring were considered, the last-week adherence percentage of the SERAD agreed with plasma drug levels in 11 of 14 cases $(78.6 \%)$, and in 8 of $14(57.1 \%)$ when the last month was evaluated. In this subgroup, pill count agreement was found in 8 of 9 cases (88.9\%). Finally, rates of last-week electronic monitoring showed agreement in 11 of 14 cases $(73.4 \%)$ and in 12 of $15(80 \%)$ for the last month.

\section{DISCUSSION}

The results of this study reveal that the SERAD questionnaire presents an accuracy comparable to other adherence measurement methods; in addition, it is a feasible, reliable, and useful instrument for assessing HAART adherence in HIV-infected patients.

The feasibility of the SERAD was based particularly on the time taken to apply the questionnaire. This variable was controlled in each form and it was observed that the SERAD is a brief and rapid assessment instrument (particularly due to the 3.7 min obtained as the mean of the total time). This advantage should also be evaluated on the basis of the correct use of the SERAD shown by participants and evaluators. In this regard, the application of the questionnaire was also monitored in order to achieve an adequate questionnaire correction process. There was no questionnaire that could not be corrected, and

FIG. 2. (A) Agreement between pill count and SERAD in last month adherence measure. Month $1(n=236)$. (B) Agreement between pill count and SERAD in last month adherence measure. Month $2(n=210)$. (C) Agreement between pill count and SERAD in last month adherence measure. Month $3(n=138)$. 
there were no mistakes limiting the obtainment of adherence measures. In addition, interviewers felt confident using the questionnaire and confirmed that it could be applied quickly and easily.

The study of the validity of a questionnaire is not an easy task. Moreover, patient self-reporting has usually been questioned, since the information provided may be biased. ${ }^{9}$ Real values may be overestimated and reliable data might be difficult to collect if the responder does not collaborate adequately. This limitation is obviously not easy to solve. Moreover, when questions cover a short time, values may not be representative, and if they cover a longer period of time, forgetfulness may produce an undesirable effect. ${ }^{21}$ For this reasons the elaboration of SERAD took these issues into account. Questions formulated within the questionnaire were open and covered three time settings, the last week, the last month, and the last 3 months, although only the first two were considered in the study. In this sense, the measure showing the greatest stability was the last-week adherence value.

Since there is no "gold standard" for comparing methods, we seek high levels of agreement when studying the outcomes of the different assessing methods. Consequently, we observed high levels of agreement between methods, specially when high levels of adherence existed; in contrast, this agreement decreased when adherence scores were lower. Probably this is a bias in the results of the study. Nevertheless, we think that this is critical for the design study, but not a limitation of the questionnaire. One reason for finding low validity assessing low levels of adherence may probably be due to the significant number of participants who dropped out of the study within 3 months. Considering this, the association between low agreement and low adherence may be the result of the relationship between being nonadherent and being more likely to drop out of care, and thus out of the study. From this point of view, the most adherent subjects would have been who would have arrived at the end of the study follow-up. Furthermore, other explanations for this low agreement may be associated with the effect of overestimation in self-reporting methods. ${ }^{9}$ In this sense, it is expected that the patient report may differ more considerably when more missed doses are reported, as found in other studies. Therefore this limitation must be clearly taken into consideration, not only for the SERAD questionnaire, but for all the studies investigating the adherence to HAART.

Finally, usefulness was assessed in terms of the advantages presented by the questionnaire. In our opinion, perhaps the main advantage of the SERAD was the possibility of obtaining different quantitative adherence percentages, providing nine measures, separated by three time settings (last week, last month, and last 3 months), and two types of adherence measures (with regard to the total number of doses prescribed and the total times that intake conditions are not observed). Similarly, an additional characteristic of the SERAD was the emphasis on associating inadequate adherence with its reasons. There is no doubt that it is important to know the reasons for presenting suboptimal adherence and, in this sense, the SERAD consequently covers each reason for each kind of inadequate behavior. The possibility of assessing intake conditions observed is another advantage of the questionnaire, and while this percentage does not appear as a resistant measure in our investigation, this aspect may be addressed more extensively in further studies.

\section{ACKNOWLEDGMENTS}

We would like to acknowledge the collaboration offered by colleagues who composed the SERAD validation team: Fundació Lluita contra la SIDA: Jose A. Muñoz-Moreno, Albert Tuldrà, Carmina R. Fumaz, Marta Barceló, Roger Paredes, Eva Romeu, Sebastià Videla. Hospital Universitari Germans Trias i Pujol: Ma José Ferrer, Bonaventura Clotet, Xavier Bonafont. Facultat de Psicologia, Universitat Autònoma de Barcelona: Tatiana Rovira, Ramon Bayés, Carme Viladrich, Isabel Izquierdo. Hospital Clínic de València: M José Galindo, Isabel Hurtado. Fundació Hospital-Asil de Granollers: Enric Pedrol, Anna Soler. Hospital Ntra Sra del Mar: Hernando Knobel, Alexia Carmona. Hospital Clínic i Provincial de Barcelona: Carles Codina, Maite Martín. Hospital Clínico de Granada: José Hernández Quero, Marisol Navas. Hospital General de Vic: Josep Vilaró, Ma Jesús Martínez. Hospital Juan Canalejo: José Pedreira, Josefina Baliñas, M ${ }^{\mathrm{a}}$ Victoria Canosa. Hospital de Palamós: Àngels Masabeu. Hospital de Donosti: José Antonio Iribarren, Garbiñe Liceaga. Consorci Sanitari del Parc Taulí: Ferran Segura, Montserrat Bernaus.

Financial support for this study was provided by Grant 2155/00 from FIPSE (Fundación para la investigación y la prevención del SIDA en España).

\section{REFERENCES}

1. Paterson DL, Swindells S, Mohr J, et al:: Adherence to protease inhibitor therapy and outcomes in patients with HIV infection. Ann Intern Med 2000;133:21-30.

2. Low-Beer S, Yip B, O'Shaughnessy MV, et al.: Adherence to triple therapy and viral load response. J Acquir Immune Defic Syndr 2000;23:360-361.

3. Bangsberg DR, Perry S, Charlebois ED, et al.: Non-adherence to highly active antiretroviral therapy predicts progression to AIDS. AIDS 2001;15:1181-1183.

4. Mocroft A, Ledergerber B, Katlama C, et al.: Decline in the AIDS and death rates in the EuroSIDA study: An observational study. Lancet 2003;362:22-29.

5. Crum NF, Riffenburgh RH, Wegner S, et al.: Comparisons of causes of death and mortality rates among HIV-infected persons: Analysis of the pre-, early, and late HAART (highly active antiretroviral therapy) eras. J Acquir Immune Defic Syndr 2006;41: 194-200.

6. Simoni J, Pearson C, Pantalone D, et al.: Efficacy of highly active antiretroviral therapy interventions in improving adherence and HIV-1 RNA viral load: A meta-analytic review of randomized controlled trials. J Acquir Immune Defic Syndr 2006;43:S23-S35.

7. Kerr T, Walsh J, Lloyd-Smith E, Wood E. Measuring adherence to highly active antiretroviral therapy: Implications for research and practice. Curr HIV/AIDS Rep 2005;2(4):200-205.

8. Berg KM and Arnsten JH: Practical and conceptual challenges in measuring antiretroviral adherence. J Acquir Immune Defic Syndr 2006;43(Suppl 1):S79-87.

9. Simoni JM, Kurth AE, Pearson CR, Pantalone DW, Merrill JO, and Frick PA: Self-report measures of antiretroviral therapy ad- 
herence: A review with recommendations for HIV research and clinical management. AIDS Behav 2006;10(3):227-245.

10. Giordano TP, Guzman D, Clark R, Charlebois ED, and Bangsberg DR: Measuring adherence to antiretroviral therapy in a diverse population using a visual analogue scale. HIV Clin Trials 2004;5(2): 74-79.

11. Beck AT, Kovacs M, and Weissman A: BDI, Beck Depression Inventory. Psychological Corp, San Antonio, TX, 1979.

12. Cattell RB: 16 PF-5 Manual. Institute for Personality and Ability Testing, Champaign, IL, 1984.

13. Hewstone M and Strobe W: Introduction to Social Psychology: A European Perspective. Blackwell, London, 2001.

14. Fundació Lluita contra la SIDA website. SERAD questionnaire [online]. Available from http://www.flsida.org/serad (Accessed 17 April 2007).

15. Oyugi JH, Byakika-Tusiime J, Charlebois ED, et al.: Multiple validated measures of adherence indicate high levels of adherence to generic HIV antiretroviral therapy in a resource-limited setting. J Acquir Immune Defic Syndr 2004;36:1100-1102.

16. Duong M, Piroth L, Grappin M, et al.: Evaluation of the patient medication adherence questionnaire as a tool for self-reported adherence assessment in HIV-infected patients on antiretroviral regimens. HIV Clin Trials 2001;2(2):128-135.

17. Godin G, Gagne C, and Naccache H: Validation of a self-reported questionnaire assessing adherence to antiretroviral medication. AIDS Patient Care STDS 2003;17:325-332.
18. Tuldrà A, Fumaz CR, Ferrer MJ, et al.: Prospective randomized two-arm controlled study to determine the efficacy of a specific intervention to improve long-term adherence to highly active antiretroviral therapy. J Acquir Immune Defic Syndr 2000;3:221-228.

19. Hugen PWH, Verweij-Van Wissen CPWGM, Burger DM, et al.: Simultaneous determination of the HIV-protease inhibitors indinavir, nelfinavir, saquinavir and ritonavir in human plasma by reversed-phase high-performance liquid chromatography. J Chromatogr B Biomed Sci Appl 1999;727:139-149.

20. Bland JM and Altman DG: Measuring agreement in method comparison studies. Stat Methods Med Res 1999;8:135-160.

21. Paterson DL, Potoski B, and Capitano B: Measurement of adherence to antiretroviral medications. J Acquir Immune Defic Syndr 2002;13(Suppl. 3):S103-106.

Address reprint requests to:

Jose A. Muñoz-Moreno Lluita contra la SIDA Foundation-HIV Unit Hospital Universitari Germans Trias i Pujol Ctra. de Canyet, $S / N$ 08916, Badalona, Barcelona, Catalonia, Spain

E-mail: jmunoz@flsida.org 